\title{
La vigencia de la responsabilidad social universitaria en el Perú: una propuesta desde el capital social
}

\author{
Javier Díaz-Albertini Figueras
}

\section{Resumen}

El artículo tiene dos partes principales. En la primera, explicaré mis iniciales dudas y recelo sobre el uso de "responsabilidad social" al referirse a las universidades, especialmente cuando reemplaza a la tradicional "proyección social". La responsabilidad social -al igual que el planeamiento estratégico, la reingeniería y el servicio al cliente- es un concepto proveniente de la empresa privada, que no siempre tiene adecuada adaptación en otras áreas de la actividad humana, en particular en aquellas que tienen como principal meta otros motivos que no son el afán de lucro. La universidad moderna siempre ha tenido como misión proyectarse hacia la comunidad -sea local, nacional o global-, mientras que la empresa recién comienza a hacerlo en forma sistemática a partir de la agenda de responsabilidad social. Es por ello que consideraba redundante que las universidades asumieran una misión (responsabilidad social) que ya venían desarrollando (proyección social). Sin embargo, los cambios en las universidades, en las últimas dos décadas -primordialmente, la creciente competencia y el incremento en su mercantilización-, han estado mellando el espíritu de extensión hacia la comunidad y disminuyendo los recursos y la energía destinados a ello. Por lo anterior, he terminado comprendiendo las bondades de la propuesta de la responsabilidad social y la necesidad de impulsarla en y desde nuestros claus- 
tros. En la segunda parte, en forma incipiente, se busca desarrollar cómo podrían contribuir las universidades a la responsabilidad social al formar y generar mayor capital social en nuestro país, especialmente al referirnos a un aumento en la confianza interpersonal e institucional y a un mayor apego a las normas.

\section{Presentación}

Confieso que por algún tiempo me parecía bastante extraño que se intentara introducir el término de responsabilidad social en el funcionamiento de las universidades. Entendía que era una temática e impulso dirigidos y limitados más bien al sector empresarial. Con ello se buscaba variar el paradigma de que las empresas respondían casi exclusivamente a la producción de bienes y servicios y la generación de lucro. En el pasado, ausente estaba la idea de que debían contribuir al mejoramiento sostenido de sus comunidades internas y externas.

Parte de mi incredulidad surgía por el hecho de que varias de las metodologías e instrumentos originados en la actividad privada con fines de lucro, habían terminado siendo adoptados acríticamente por las universidades y otras organizaciones no orientadas primordialmente hacia igual finalidad. Tal era el caso de conceptos y estrategias que habían nacido en el mundo empresarial, como el planeamiento estratégico, el liderazgo en la gestión, la reingeniería, la gestión orientada a la atención al cliente, la calidad medida de acuerdo al ISO, el outsourcing y tercerización, y muchos otros más. Como sociólogo me maravillaba ver cómo el paradigma y la hegemonía liberal -especialmente, sus concepciones de la competencia y del mercado- habían invadido las principales organizaciones e instituciones, desde los municipios rurales hasta los centros de educación superior. Muchas de estas incorporaciones de estrategias empresariales no acababan de adaptarse bien a las necesidades de organizaciones públicas o sin fines de lucro, que, a pesar de producir bienes y servicios, tenían orientaciones variadas y diferentes de las de la empresa privada.

Por ejemplo, en algunas universidades comenzaron a referirse al alumno como "cliente", en un intento por acercar estrategias de atención, fidelización y mercadeo al ámbito de la educación superior. Sin embargo, la relación comercial del alumno con la universidad y sus docentes es bastante disímil a la del comprador de un auto, una refrigeradora o una hamburguesa; de igual manera que la relación entre un 
médico y un paciente (no "cliente") conlleva mucho más que el pago de un servicio. En los casos de la educación y salud, se está apostando por una relación de mediano a largo plazo, cuyos resultados no pueden medirse inmediatamente, sino en un proceso que puede tomar toda la vida. Al comprar un auto, por el contrario, tengo en mente varias especificaciones que atienden a mis necesidades actuales y de un futuro bastante cercano. Por ejemplo, cuántos somos en mi familia en este instante, los usos que le daré en el corto plazo y con cuánto dinero cuento ahora. Y sé bien que estas decisiones son de corto plazo porque en pocos años me compraré un auto nuevo que responderá a nuevas necesidades y expectativas.

Más que consumir educación y salud, en el fondo invertimos en ellas. Como toda inversión, lo hacemos con la esperanza de un retorno. En el caso de la educación, su retorno tiene incidencia directa en la calidad de vida de la persona, ya que marcará los ingresos que percibirá durante su trayectoria laboral. Al invertir en educación, se está asumiendo un riesgo de largo plazo, ya que los resultados se verán años después de haberla recibido. Es cierto que puedo cambiar lo que he estudiado o la carrera elegida si la originalmente seleccionada no es "rentable", pero el costo de hacerlo es sumamente alto. La educación también sigue jugando un rol importante en las posibilidades de movilidad social y en la justificación de las diferencias de ingresos en una sociedad. Como bien ha demostrado Yamada (2007), en el Perú todavía vale la pena la inversión en la educación (especialmente, la superior), ya que sigue existiendo una relación directa y robusta entre el número de años estudiados y el ratio de retorno económico.

Debido a que el estudiante y sus padres normalmente no tienen una idea clara y detallada de lo que implica una formación apropiada para culminar la carrera escogida, dependen fuertemente de los criterios establecidos por cada universidad, es decir, por sus profesores y profesionales, o sea, quienes diseñan la malla curricular. Existe una situación clara de asimetría de información. La falta de información por parte del estudiante tiende a ser compensada mediante la búsqueda de un proveedor confiable. Más aún si la educación es en una institución privada financiada por los padres, ya que se tiende a dar lo que algunos economistas denominan el "fracaso de contrato", pues el adquiriente del servicio (el padre y la madre) no es el que lo consume (sino el hijo o hija), y ello dificulta la evaluación de la calidad. Según Hansmann (2003), en estos casos funciona la "hipótesis de la confianza", es decir que ante la falta de 
información y criterios para evaluar el servicio educativo, se busca contratar operadores confiables y escrupulosos. Por este conjunto de razones, resulta difícil encasillar al estudiante bajo el mote de "cliente".

Sin embargo, mayor era mi extrañeza en el caso de la responsabilidad social. Después de todo, podía comprender que la universidad incorporara algunos mecanismos y estrategias de la empresa que habían mostrado resultados, trátese del área de la gestión de recursos humanos, en el planeamiento, o en la medición y evaluación de resultados. Pero en el caso de la responsabilidad social, mi idea era que las universidades, en todo caso, tenían que enseñar a las empresas privadas, y no al revés. Después de todo, el sector empresarial está orientado al mercado, a la búsqueda del mayor beneficio económico posible para sus propietarios e inversionistas. Es decir, es un espacio en el cual reina el egoísmo racional.

Como buen egoísta racional, el empresario siempre ha reconocido que para lograr mejor su fin de lucro, hay algunos elementos de organización social que son indispensables. Lo "racional" de su conducta egoísta era justo lo que lo acercaba -aunque en forma limitada- a los aspectos más sociales de su actividad económica. De ahí la aceptación de la necesidad de reglas de juego para el intercambio comercial (confianza, seguridad jurídica), lo cual también justificaba la existencia de un Estado de derecho y la tributación para su mantenimiento, la búsqueda de calidad de los productos y la responsabilidad ante los consumidores como mecanismo para generar fidelidad en una marca y asegurar la demanda, y la aplicación de políticas de recursos humanos que permitieran captar a los más capaces y mantenerlos en el empleo, contribuyendo así a la equidad de oportunidades, y hasta la toma de cierta conciencia de su entorno y la necesidad de no contaminarlo en demasía.

Antiguamente, la responsabilidad empresarial tendía a reducirse a respetar el sistema normativo y jurídico que establecía los marcos, las regulaciones y los controles a la conducta egoísta y que buscaba construir el bien común. En forma creciente, además, a partir del siglo XX, el sector empresarial también reconoce que el Estado es el encargado de asegurar no solo los derechos civiles y políticos, sino también los socio-económicos como la educación y la salud, y crear condiciones propicias para la generación de empleo. La contribución empresarial a estos procesos era la tributación. Dentro de esta perspectiva, la actuación y contribución empresarial en aspectos sociales se limitaba al pago 
de impuestos y al cumplimiento de las normas de cada localidad. En términos políticos, se reducía a buscar, vía el cabildeo y la presión de grupo, las condiciones más propicias para el funcionamiento del mercado y la promoción de su rubro productivo. Para ello, se organizaba en gremios empresariales según el rubro de producción e intereses.

El cimiento sobre el cual se construye esta supuesta delimitación de las esferas de actuación de las empresas y del mercado en la sociedad, tiene que ver con la filosofía y las teorías económicas liberales que postulaban que el eficiente desempeño económico solo era posible con su independencia. El buen funcionamiento del mercado, se decía, debía responder a mecanismos propios de la oferta y demanda, procesos que para desarrollarse correctamente dependían de la libertad económica de los individuos y la mínima intervención de los otros sectores de la sociedad (Estado-sociedad). Estos mismos planteamientos postulaban que la sociedad estaba compuesta por tres sectores (el mercado, el Estado y la sociedad) y cada uno tenía sus propias leyes de funcionamiento. Es por ello, como bien plantea Wallerstein (1996), que se crean tres ciencias sociales, una por cada sector de la sociedad, y que llegaron a ser conocidas como la economía (mercado), las ciencias políticas (Estado) y la sociología (sociedad).

Algunas empresas y sus propietarios, a través de donaciones filantrópicas, buscaban incidir sobre problemas específicos de sus respectivas sociedades. El altruismo empresarial ha sido analizado en varios países, incluyendo el nuestro, y con frecuencia tomaba la forma de fundaciones empresariales o familiares que apoyaban actividades culturales o de apoyo social (Portocarrero, 2000). Hay múltiples estudios intentando explicar esta conducta altruista, especialmente en Estados Unidos, sociedad que tiene una larga y amplia experiencia en la filantropía empresarial e individual ${ }^{1}$. La filantropía, sin embargo, casi siempre se financiaba con recursos residuales de la actividad económica, los cuales dependían directamente del desempeño empresarial en el mercado. En cambio, la responsabilidad social, como veremos más adelante,

1 Incluso se diferencia entre el altruismo "puro" e "impuro". En el primero, el donante no recibe ningún beneficio privado de su actividad, mientras que en el segundo sí. Es difícil imaginarse, sin embargo, un altruismo plenamente puro, porque parte de los beneficios puede ser individual, es decir, el donar genera un sentimiento de bienestar y "calidez" (Rose-Ackerman, 1996). 
por definición tiene que ser sostenible y forma parte de las operaciones usuales de la empresa.

Por factores que han sido analizados por muchos autores, las empresas comienzan a ampliar su visión acerca de la responsabilidad que les compete, al operar en una comunidad o sociedad. Esto se debe a factores que oscilan desde los cambios en la conducta de los consumidores (mayor preocupación sobre las condiciones de producción, mayor conciencia en la salud), la mayor conciencia del impacto en el medio ambiente y la sostenibilidad de las actividades económicas, la presión social con respecto a la ciudadanía empresarial y su apoyo al ejercicio y protección de los derechos humanos, el efecto perverso de la corrupción y la conducta no ética en el mundo de las relaciones sociales y económicas (reducción de capital social), hasta problemas de calidad y producción de sus trabajadores debido a condiciones en el ámbito de trabajo, entre muchos otros (Comisión de las Comunidades Europeas, 2001).

Estos y muchos otros problemas llevaron a una creciente preocupación por las relaciones sociales al interior y al exterior de la empresa, al efecto que tienen sobre las operaciones económicas y la calidad de vida general. Es decir, surge la idea de que la empresa debe servir a la sociedad y que esto no solo se cumple con la eficiente producción de bienes y servicios y empleos, sino que también debe ocurrir dentro de un entorno ético, es decir, de responsabilidad. Se va forjando así un movimiento global que impulsa a la responsabilidad social empresarial (RSE) y que se plasma en diversas organizaciones, documentos y hasta en un pacto mundial impulsado por las Naciones Unidas 2 .

La Comisión de las Comunidades Europeas (CCE) define esta orientación de la siguiente manera:

La mayoría de las definiciones de la responsabilidad social de las empresas entienden este concepto como la integración voluntaria, por parte de las empresas, de las preocupaciones sociales y

2 Desde noviembre del 2003 funciona el Pacto Global-Perú, y desde el 2005 es gestionado por la Confederación Nacional de Instituciones Empresariales Privadas (Confiep). Según los datos de la página web de la Confiep, son 113 empresas peruanas suscritas (véase http://www.confiep.org.pe/articulos/gestion-institucional/5el-pacto-mundial-en-el-peru). 
medioambientales en sus operaciones comerciales y sus relaciones con sus interlocutores. (2001, p. 4)

Mientras que para el Consejo Mundial Empresarial para el Desarrollo Sostenible (WBCSD)...

La responsabilidad social corporativa es el compromiso permanente de los negocios de comportarse éticamente y de contribuir al desarrollo económico al tiempo que se mejora la calidad de vida de los trabajadores y sus familias, de la comunidad local y de la sociedad en general. (Holme y Watts, 2000, p. 6)

La universidad, al contrario, había evolucionado durante el siglo XX hacia organizaciones que tenían como sus principales objetivos la calidad de los productos y servicios ofrecidos a la comunidad académica, pero también a la sociedad en general. Esto, por ejemplo, se capta con meridiana claridad cuando vemos cómo la universidad en el Perú, bajo su concepción moderna, ha estado -o debería haber estado- íntimamente ligada con un compromiso con el desarrollo personal y social de sus integrantes, y a la vez con una proyección social hacia la comunidad como medio para contribuir al desarrollo nacional. Esto es clarísimo en la Ley 23733 de 1983, que en su primer artículo sobre los fines de la universidad, señala, en los incisos c y d:

c) Formar humanistas, científicos y profesionales de alta calidad académica, de acuerdo con las necesidades del país, desarrollar en sus miembros los valores éticos y cívicos, las actitudes de responsabilidad y solidaridad social y el conocimiento de la realidad nacional, así como la necesidad de la integración nacional, latinoamericana y universal;

d) Extender su acción y sus servicios a la comunidad, y promover su desarrollo integral [...]

Resulta interesante notar y subrayar los términos "necesidades del país", desarrollar en sus miembros "valores éticos y cívicos", actitudes de "responsabilidad y solidaridad social", y, en términos del entorno, la proyección de sus acciones y la promoción del desarrollo integral. En la nueva y recientemente promulgada ley de universidades (Ley

3 La traducción es mía. 
30220), se mantienen y amplían estos principios, incluyéndose aspectos como "Plurarismo, diálogo intercultural e inclusión" (5.8), "Pertinencia y compromiso con el desarrollo del país" (5.9) y "Pertinencia de la enseñanza e investigación con la realidad nacional" (5.15). Como fines, se incluye formar a profesionales de alta calidad y con pleno sentido de responsabilidad social (6.2), la proyección hacia la comunidad para promover su cambio y desarrollo (6.3), y promover el desarrollo humano y sostenible en el ámbito local, regional, nacional y global (6.6), entre otros factores que sostienen una presencia e incidencia de la universidad en la sociedad. Inclusive, la nueva ley incluye un capítulo (XIII) titulado "Responsabilidad social universitaria", que además de definirla, señala que se dedicará el $2 \%$ como mínimo del presupuesto para sus fines.

Esto marca una clara diferencia con respecto al sector empresarial, ya que la responsabilidad social no es un aspecto nuevo del quehacer universitario, es una práctica que ha estado normada mucho antes de la evolución de la preocupación actual. En todo caso, la nueva ley recoge esta preocupación y la adapta a las actuales conceptualizaciones. Entonces, ¿por qué hemos de preocuparnos por construir una noción de "responsabilidad social universitaria" (RSU) cuando siempre ha estado presente como finalidad básica de los estudios superiores?

La principal razón es que, a pesar de las buenas intenciones, la comunidad universitaria no ha cumplido cabalmente con estas finalidades. Como enfatizan varios estudiosos, muchos de ellos vinculados a RSU, los tiempos también han cambiado para las universidades y en los últimos veinte años han perdido de vista estos fines tan claramente presentados en la antigua y la nueva ley, a pesar de ser exigidos por la ciudadanía y ser parte integral de los estatutos de las universidades en el país.

Vallaeys, de la Cruz y Sasia (2009), por ejemplo, consideran que es un prejuicio plantear que la responsabilidad social es una invasión de la universidad por parte de la lógica empresarial, porque en su versión "universitaria" es más bien una estrategia que desarrolla finalidades, estrategias, acciones y proyectos orientados a mejorar los impactos de la propia misión universitaria: cognitivos, epistemológicos y sociales. Para estos autores, la RSU no es un proceso que mercantiliza a la universidad, sino más bien que enfatiza su compromiso ético con los profesores, estudiantes y personal administrativo, creando una comunidad de enseñanza-aprendizaje a la vez que consolida (¿o inicia?) un trabajo de 
reflexión con el resto de la sociedad, buscando incidir en temas centrales como la justicia social, el desarrollo y la sostenibilidad ambiental.

Desafortunadamente, en muchas universidades, en las últimas dos décadas, las preocupaciones han estado limitadas a captar alumnos, a impartir enseñanzas a un público estudiantil masificado, a enfrentar restricciones presupuestarias en su frente interno y a limitar sus acciones de extensión o proyección social en su frente externo. Su finalidad y misión se han ido diluyendo debido a diversos factores del entorno -que a continuación mencionaré brevemente-, o simplemente han sido obviadas o ignoradas. ¿Cuáles han sido estos factores? He aquí los que considero esenciales:

a. Como bien indica Claudio Rama (2006) en su análisis de la evolución de las reformas universitarias latinoamericanas, nuestras instituciones han tenido dos grandes reformas desde la segunda década del siglo XX. La primera nace con la famosa Reforma de Córdova de 1918, que impulsó la autonomía universitaria, las formas de cogobierno, la gratuidad de la enseñanza, la ampliación hacia carreras profesionales, técnicas y científicas, y la limitación de la incidencia del Estado al soporte financiero. Este modelo privilegiaba la educación superior pública. En el caso del Perú, se refleja con claridad en el hecho de que para 1960, existían nueve universidades en el país y solo una era privada. La segunda reforma, según Rama, que en el caso del Perú comienza a producirse en los años sesenta, es cuando la educación universitaria pasa a ser un sistema "binario", debido a la creciente presencia de la universidad privada como producto del incremento de la demanda -como resultado de la ampliación de la educación escolar secundaria y las exigencias del mercado de trabajo- y a limitaciones presupuestarias del Estado como producto del estancamiento económico que se manifiesta con total claridad a mediados de los años setenta. Esto conlleva varios factores que no pretendemos discutir en el presente documento, pero quizás el más importante fue la enorme oferta de estudios superiores, muchas veces sin las condiciones básicas de calidad y guardando poca relación con las necesidades reales de las oportunidades y necesidades del mercado de trabajo.

b. La competencia por los alumnos, hecho que no ocurría en la etapa anterior, se da actualmente en todos los sentidos. Las 
públicas compiten entre sí y con las privadas y entre las primeras y las segundas. Como parte de la competencia se amplía la oferta de carreras al intentar captar más postulantes y estudiantes. A su vez, carreras no consideradas "rentables" comienzan a ser sacrificadas a pesar de su importancia en el desarrollo humanista y ético de la sociedad. Esto se exacerba con el Decreto Legislativo 882 de noviembre de 1996, que por primera vez permite que la universidad se constituya como empresa privada con fines de lucro. Resulta impresionante observar el despliegue de recursos universitarios para el mercadeo, la publicidad, la llegada a los potenciales postulantes y el peso que se está dando a las estrategias de "imagen institucional". Todo esto, no obstante, para captar a estudiantes que aún no tienen una idea clara de lo que deben buscar al momento de decidir por una educación universitaria. Es así que con frecuencia se aplican técnicas de publicidad que no enfatizan en los estudios, el esfuerzo, la investigación y el compromiso con el país. Por el contrario, se hace hincapié en la infraestructura, en la rápida profesionalización y la llegada al mercado de trabajo. Esto no significa que esté en contra de la competencia entre los claustros universitarios, sino que se ha desviado de asuntos centrales que caracterizan a la universidad, como son los logros científicos, la preparación de sus recursos humanos, la búsqueda de la calidad y el aporte al desarrollo nacional.

c. En sesenta años ha cambiado totalmente la configuración de la universidad peruana. De un sistema dominado por la universidad pública, hemos pasado a un sistema tripartito en el cual la universidad-empresa es la que muestra mayor dinamismo y crecimiento $^{4}$. Según la Dirección de Estadística de la Asamblea Nacional de Rectores, en 2012, los alumnos matriculados en la universidad privada (642 203) ya duplicaban a los de la pública (321 581) $)^{5}$. Este explosivo incremento de las universidades no ha estado acompañado de mecanismos de regulación y acreditación,

4 http://www.anr.edu.pe/files/directorio_2014_web_18_09_2014_correos.pdf

5 Es posible consultar estas estadísticas en la página web del Instituto Nacional de Estadística e Informática, en la siguiente dirección: http://www.inei.gob.pe/ estadisticas/indice-tematico/sociales/. 
lo cual ha generado una oferta inescrupulosa y la denominación de "universidades chatarra" o "de medio pelo" a muchos de los centros creados en los últimos años 6 .

d. La calidad en sus fines de formación, investigación y aporte al desarrollo nacional ha decaído, a su vez, por otras razones:

La mayoría de los profesores son contratados a tiempo parcial, como medida de disminución de costos. Bajo estas condiciones, no tienen tiempo ni posibilidad de participar plenamente en la comunidad universitaria, preparar con detenimiento sus clases y mantenerse actualizados. En el análisis que sustenta el proyecto de la ley universitaria, hacen referencia a la situación laboral de los profesores. En las universidades públicas, 34788 profesores eran contratados y solamente 20486 eran ordinarios. Esta relación es peor en las universidades privadas. Por ejemplo, en la Pontificia Universidad Católica el 77 \% era contratado; en la Universidad San Martín de Porres, el 95 \%; y en la universidad más grande del Perú, Alas Peruanas, el $98 \%$.

Las universidades se han convertido -en su gran mayoría- en teaching colleges, una opción casi exclusiva hacia la docencia, dejando de lado la investigación. Es así que se dificulta la importante retroalimentación con el proceso de enseñanza y la búsqueda de respuestas a las problemáticas sociales más importantes.

Muchas universidades están impulsando una "sobreespecialización" en sus currículos, perdiendo de vista su esencial misión en la formación humanista. Ello, a pesar de ser un elemento primordial para desarrollar los tres aspectos esenciales de toda educación superior. Primero, las preguntas con respecto al significado que concierne la interpretación de datos, evidencias y textos. Segundo, las pesquisas sobre los valores en una enorme variedad de quehaceres: culturales, estéticos, sociales, científicos y políticos. Tercero, con respecto a la trascendencia que genera preguntas sobre la representación, en el

6 Inclusive el presidente de la República, en 2013, exhortó a los jóvenes a que no fueran engañados: “Muchas están engañando a los jóvenes, haciéndoles creer que les van a cambiar la vida. Jóvenes, eso es mentira, los están estafando. Vayan a universidades serias, no vayan a esas universidades de medio pelo cuyo título no sirve para nada. Solamente se han dedicado a hacer plata". http://peru21.pe/ politica/universidades-medio-pelo-ley-universitaria-ranking-empresas-no-egresados-humala-2191498. 
sentido de explicar y aprehender (descripción y generalización). La formación humanista es lo que contribuye a generar un sentido ético y una capacidad de interpretación reflexiva de la realidad, mientras que la sobreespecialización tiende a obviar la formación holística en favor de una preparación técnica o profesional que pierde relación con las principales necesidades y problemas del país.

La competencia, la falta de recursos, la poca presión social con respecto a su desempeño, la falta de transparencia de las mismas universidades, entre otros factores, han llevado a que estas concentren cada vez más recursos para promover su imagen dirigida a captar alumnos, en vez de dedicar más energía hacia la responsabilidad social, que está tan bien establecida en las normas e inclusive en sus propios estatutos.

De ahí que, a pesar de mis resistencias iniciales, considero que sea totalmente necesario y relevante que enfaticemos la responsabilidad social, y establezcamos mecanismos institucionales e interinstitucionales para encarar sus retos. En este sentido, el concepto de responsabilidad social es importante, no solo por la rigurosidad de su definición, sino también como factor "sensibilizador". Entiendo la sensibilización como un mecanismo mediante el cual las personas e instituciones:

- Toman conciencia de la magnitud de una situación, experiencia, vivencia.

- Adquieren la capacidad de percibirla (literalmente, descubrirla), impidiendo su invisibilización.

- Se comprometen emocionalmente con ella.

- Tienen a su disposición capacidades y mecanismos para actuar.

Podemos asumir, entonces, que cuando sensibilizamos estamos generando una vocación de descubrir, de adquirir conocimientos nuevos y alimentar las posibilidades de acción. La sensibilización es un paso esencial para que la comunidad universitaria actúe responsablemente ante sus stakeholders y la sociedad en general.

\section{La universidad y el capital social}

Según Vallaeys (2006), la responsabilidad social es una gerencia ética e inteligente de los impactos que genera una organización en su entorno humano, social y natural. Es ética porque todos los potenciales afectados por la actividad de la organización deberán poder retirar los mayo- 
res beneficios y sufrir los menores daños de ella. Es inteligente porque las actividades realizadas deben reportar un retorno en beneficios a la organización, recursos que posibilitan la sostenibilidad de las operaciones con sentido de responsabilidad.

En el caso de la organización universitaria, Vallaeys reconoce cuatro impactos, a saber:

(1) Impactos de funcionamiento organizacional: el impacto sobre su personal administrativo, los docentes y la población estudiantil, al igual que los efectos que tienen sus operaciones en el medio ambiente.

(2) Impactos educativos: mediante la formación de jóvenes y profesionales, incluyendo la dimensión de compromiso ético y solidaridad.

(3) Impactos cognitivos y epistemológicos, vía la generación de conocimientos.

(4) Impactos sociales, no solamente en la formación, sino como referente y actor social que debe promover el progreso, el capital social y difundir conocimientos, metodologías y estándares éticos de conducta entre nuestros ciudadanos.

Como ejercicio intelectual, plantearé un ejemplo de cómo las universidades pueden desarrollar su responsabilidad social según el impacto que Vallaeys denomina precisamente "social", específicamente en lo que concierne a la generación y fortalecimiento del capital social. Podríamos definir este tipo de capital como "las características culturales y de la estructura social que facilitan la colaboración, la cooperación y la acción colectiva entre individuos, grupos, organizaciones e instituciones" (Díaz-Albertini, 2003). En forma creciente, analistas y estudiosos han enfatizado que este capital -en conjunto con el productivo, el natural y el humano- es uno de los requisitos para fomentar el progreso y el desarrollo humanos. ¿Cómo contribuye el capital social al funcionamiento de la sociedad y al desarrollo? Las formas principales son:

- Reduce la incertidumbre y los costos de transacción.- En toda relación social existe un intercambio (transacción). A mayor confianza en una sociedad, el riesgo de incumplimiento disminuye. En sociedades con poca confianza, las transacciones son muy costosas porque siempre existe mayor riesgo de ser engañado, traicionado o estafado. De ahí que los individuos busquen diversas formas de protegerse contra la incertidumbre. Estudios a nivel internacio- 
nal, por ejemplo, muestran que los niveles de confianza interpersonal e institucional están directamente relacionados con el ritmo del crecimiento económico (Knack y Keefer, 1997).

- Facilita la coordinación para producir todo tipos de bienes.- Casi toda producción involucra la intervención de varios actores. Esto es esencial para la producción de bienes públicos, semipúblicos y privados.

- Reduce las relaciones autoritarias e incentiva la participación social.Diversos estudios muestran que a mayor sociabilidad (participación, colaboración, cooperación), mayor democracia, especialmente en la tradición teórica de Robert Putnam (1994).

En términos específicos, el capital social serían los beneficios sociales a los que una persona o grupo social pueden acceder por el hecho de ser parte de ciertas redes sociales y estructuras (Portes, 2000). Si vivo en una comunidad en la cual las personas respetan las normas y, por ejemplo, cumplen con el reglamento de tránsito, me veré beneficiado de diversas maneras. Mis hijos podrán jugar en la calle y montar bicicleta, favoreciéndose así su desarrollo social y sentido de comunidad; existirán menos rompemuelles y no sufriría tanto la dirección y amortiguación del auto, disminuyendo los costos de mantenimiento, reparación y reemplazo; habrá menos accidentes y disminuirán las primas de los seguros vehiculares, de salud y vida. Es un beneficio generado socialmente, pero que puede ser apropiado de forma individual.

Hay una tendencia a coincidir sobre los tres factores que contribuyen a crear capital social (Díaz-Albertini, 2010):

(a) Los niveles de confianza interpersonal e institucional, porque predisponen a las personas a relacionarse con los demás.

(b) La legitimidad y efectividad de las normas sociales, porque facilitan las relaciones entre las personas.

(c) La densidad de las redes sociales, porque muestran los niveles de acceso de las personas a los recursos que están en control de los demás miembros de la sociedad.

Las universidades tienen mucho que aportar para el fortalecimiento de estos tres factores. Pero, por cuestión de tiempo y espacio, solo abordaré su posible contribución en lo referente a la confianza. 


\section{La universidad y la generación de confianza}

La confianza es la creencia en que los demás se comportarán de acuerdo a nuestras expectativas (Díaz-Albertini, 2010). La falta de confianza es una de las principales debilidades de la sociedad peruana. La institución World Value Surveys (WVS; Estudio Mundial de Valores, en español) ha medido en cuatro ocasiones los niveles de confianza interpersonal en el Perú y en otros cincuenta o más países ${ }^{7}$. En el cuadro que sigue presentamos los resultados.

La confianza interpersonal en el Perú y el promedio mundial (en \%)

\begin{tabular}{lcccc}
\hline & \multicolumn{4}{c}{ "Olas" de encuestas - Estudio mundial de valores (WVS) } \\
\hline & $1995-1998$ & $1999-2004$ & $2005-2009$ & $2010-2014$ \\
\hline $\begin{array}{l}\text { Confianza } \\
\text { interpersonal Perú }\end{array}$ & 4,9 & 10,6 & 6,2 & 8,4 \\
$\begin{array}{l}\text { Confianza } \\
\text { interpersonal promedio } \\
\text { en encuesta }\end{array}$ & 24,0 & 26,9 & 25,4 & 24,5 \\
$\begin{array}{l}\text { Número de países } \\
\text { participantes }\end{array}$ & 56 & 51 & 58 & 52 \\
$\begin{array}{l}\text { Posición Perú/resto del } \\
\text { mundo }\end{array}$ & $56 / 56$ & $48 / 51$ & $55 / 58$ & $46 / 52$ \\
\hline
\end{tabular}

Fuente: http://www.worldvaluessurvey.org/WVSOnline.jsp, elaboración propia.

Observamos que en un período de veinte años, el país ha mostrado niveles bastante bajos de confianza interpersonal: solo un promedio de $7 \%$ de la población afirma que se puede confiar en la mayoría de las personas, cuando el promedio mundial ha oscilado entre 24 y $27 \%$. Comparado con el resto de países participantes en las diversas muestras (con un promedio de 55 países), Perú ha ocupado los últimos lugares del ranking, e incluso en una de las "olas" ocupó el último lugar. Encontramos resultados parecidos en las encuestas anuales de Latinobarómetro, en las cuales se nos compara con otros 16 o 17 países de la región.

7 Las encuestas se realizan en "olas" (waves), debido al gran número de países involucrados. Por el momento han existido seis olas correspondientes, y el Perú ha participado en las últimas cuatro. 
Confianza interpersonal Perú - Latinobarómetro 1996-2011, en \%

$\begin{array}{llllllllllllll}1996 & 1997 & 1998 & 2000 & 2001 & 2002 & 2003 & 2004 & 2006 & 2007 & 2008 & 2009 & 2010 & 2011\end{array}$

Se puede

confiar en la

mayoría de las

personas

Puesto Perúl

total de países

$\begin{array}{lllllllllllllllllllll}14 / 17 & 14 / 17 & 14 / 17 & 14 / 17 & 13 / 17 & 12 / 18 & 10 / 17 & 11 / 18 & 10 / 18 & 12 / 18 & 16 / 18 & 15 / 18 & 16 / 18\end{array}$

Notamos una sorprendente continuidad en los resultados a pesar de los cambios políticos, económicos y sociales vividos por el país. Los niveles bajos de confianza casi siempre nos ubican, además, en el tercio inferior de los 17 o 18 países participantes en la encuesta. A esto tendríamos que añadir la inmensa desconfianza hacia las instituciones. En el Latinobarómetro del 2011, obtuvo los porcentajes más bajos de confianza en las instituciones públicas y políticas. El país se encuentra, entonces, sumido en una desconfianza generalizada hacia los demás y sus instituciones:

La desconfianza en el Perú se basa en la desconfianza de cada cual con cada cual, la baja confianza en las instituciones no es sino el reflejo de ello. Es una sociedad que no tiene resueltas, al igual que la mayoría de nuestras sociedades, cosas fundamentales que se traslucen en la manera como nos relacionamos el uno con el otro: la igualdad ante la ley, la justicia distributiva, la justicia, el uso del poder. El problema de nuestras sociedades al final del día no es la confianza, sino la desigualdad; en la medida en que podamos desmantelarla, aumentará la confianza que tenemos en el prójimo. (Latinobarómetro, 2012, p. 19)

Finalmente, a este sombrío paisaje tendríamos que añadir las percepciones que tenemos sobre el cumplimiento de las leyes. En el Latinobarómetro del 2011, solo el $12 \%$ de los encuestados manifestaron que los ciudadanos cumplían "mucho" o "bastante" la ley, el resto consideraba que se cumplía poco o nada. Ocupamos el último lugar en la región. Parecido fue el resultado cuando se les preguntó si éramos conocedores de nuestras obligaciones y derechos. Para el $17 \%$ de los peruanos, cono- 
cíamos "mucho" o "bastante"; el resto consideraba que conocíamos "poco o nada". Este resultado también nos hizo ocupar el último puesto en la región.

Sobre la base de estos y otros resultados (Díaz-Albertini, 2010), llegamos a la conclusión de que la confianza tiende a ser restringida hacia la familia y las relaciones sociales más cercanas (amistad, barrio, paisano). Siguiendo los criterios de Fukuyama (1996), esto nos hace una sociedad de confianza familista, ya que está marcada por una visión particularista de la sociedad y apegada a condiciones que en la sociología denominamos "adscritas" o "adjudicadas". Esto es diferente en las sociedades de confianza generalizada, en la cual el mismo hecho de pertenecer a una comunidad o sociedad lleva a la percepción de que los demás son confiables. Según Fukuyama, las sociedades de confianza familista tienen dificultades para construir organizaciones económicas complejas, a lo cual yo añadiría organizaciones políticas o acciones colectivas complejas. El tercero "extraño" es visto con recelo y con la idea de que nos hará algún tipo de daño, resultando difícil establecer relaciones colaborativas con los no cercanos.

En este mar de suspicacias, no obstante, las universidades destacan por gozar de uno de los niveles más altos de confianza entre las instituciones del país, si tomamos en consideración encuestas realizadas en los últimos años. Las universidades privadas tienen los niveles más altos de confianza entre las instituciones de la sociedad civil (detrás de la Iglesia católica), y la universidad pública se encuentra en los niveles más altos entre los organismos públicos e instituciones estatales. Los ciudadanos reconocen en ellas calidad, eficiencia, eficacia y honestidad en sus áreas de competencia. En una encuesta realizada por la Universidad de Lima en 2007, observamos las siguientes cifras:

Confianza en las universidades

\begin{tabular}{ccc}
\hline ¿Confía? & Universidad pública & Universidad privada \\
\hline Sí & 72,9 & 72,7 \\
No & 25,8 & 23,8 \\
NS/NR & 1,3 & 3,5 \\
TOTAL & 100,0 & 100,0 \\
\hline
\end{tabular}


Al mismo tiempo, un sector importante de la población limeña señala que el rol que cumplen la universidad pública y la privada en el desarrollo del país, se encuentra entre "muy importante" e "importante", con menos del $20 \%$ de los encuestados clasificándola como "poco" o "nada" importante.

\section{Evaluación del rol que cumplen las universidades}

\begin{tabular}{lcc}
\hline Rol que cumple & Universidad pública & Universidad privada \\
\hline Muy importante & 15,9 & 10,7 \\
Importante & 66,3 & 70,8 \\
Poco importante & 14,6 & 14,6 \\
Nada importante & 1,0 & 1,5 \\
NS/NC & 2,1 & 2,4 \\
TOTAL & 100,0 & 100,0 \\
\hline
\end{tabular}

¿Cómo puede la universidad sacar provecho de la alta estima que le otorga la ciudadanía para promover mayores niveles de confianza en el resto de la sociedad? Para ello tendríamos que ver brevemente cuáles son los factores que contribuyen a la confianza y cómo la universidad puede impactar sobre ellos:

- Un primer factor que contribuye a fortalecer la confianza es contar con creencias e ideologías comunes. En sociedades tradicionales, nacían de la convivencia en una misma comunidad, clan o familia. En sociedades modernas, tienden a estar reflejadas en intereses, ideologías y propuestas políticas o religiosas. Al compartir "visiones del mundo", nos acercamos a los demás y se puede inspirar un compromiso colectivo. Coleman (1994), uno de los principales teóricos del capital social, considera que:

Un factor que tiene efecto en la creación o destrucción de capital social es la ideología. Una ideología puede crear capital social al imponer sobre el individuo que la comparte, la demanda de que actúe en el interés de algo o alguien que no sea uno mismo. (1990, p. 320) ${ }^{8}$

8 La traducción es mía. 
Al examinar diversas experiencias de relaciones entre el sector público y la sociedad civil, Evans (1996) enfatiza la importancia de la transformación de las "visiones del mundo" que las comunidades de base y la ciudadanía tienen de sí mismas y de los demás, para poder construir las estructuras sociales conducentes al cambio social. Estos cambios en identidades e intereses normalmente provienen de la extensión y ampliación de las relaciones horizontales y verticales de las comunidades, de la información y del conocimiento que provienen desde el exterior, y de la consecuente construcción de nuevas experiencias e intereses.

Es por estas razones que es imprescindible, para enriquecer la confianza (y el capital social), comprender y apuntalar la necesaria e impostergable relación de la población, y especialmente los excluidos, con sectores de intelectuales y técnicos capaces de alimentar, apoyar y facilitar sus visiones y acciones colectivas; sobre todo cuando se busca fomentar cambios en las formas de pensar y en las prácticas, para que contribuyan en la sostenibilidad de la acción colectiva.

En nuestro país, la mayoría de la población no cuenta con el tiempo o recursos necesarios para desarrollar estrategias, técnicas y mecanismos de organización y acción, ya que para ellos el tiempo es su único aliado en la supervivencia. En un mundo de grandes transformaciones, los más pobres con frecuencia temen a los cambios porque no tienen los mecanismos o conocimientos para hacerles frente. El tiempo para diseñar programas para el futuro y opciones viables -incluyendo propuestas de gestión organizativa y política-, corresponde a los intelectuales y técnicos, sean del sector público o privado. El gran reto, como han notado tantos analistas, es que estas propuestas puedan ser discutidas y contrastadas con las opiniones y propuestas de los excluidos, sin imposiciones y mediante un diálogo horizontal. De esta capacidad, difícil mas no imposible, de implementar en la práctica, es que dependen las posibilidades de enriquecer la confianza de los pobres en un futuro alternativo y en un orden social diferente. Y una de las ideas fuerza que debemos impulsar desde los claustros universitarios es la fundamental importancia de la institucionalidad democrática.

En el 2006, el Programa de Naciones Unidas para el Desarrollo (PNUD) realizó una ambiciosa encuesta en el país para analizar las 
concepciones y sentimientos acerca de la democracia ${ }^{9}$. Los resultados confirman que existe un nivel muy alto de desconocimiento acerca de la democracia y la desconfianza hacia el sistema político nacional. Al interrogar qué responderían a la pregunta "¿qué es la democracia?", el 34,9\% dijo que respondería que no sabía o que no tenía idea; el 26,5 \% dijo que tenía una idea pero que no le importaba. El estudio resalta que este desconocimiento y desinterés aumentaba en porcentajes superiores al $50 \%$ entre los pobres, campesinos, indígenas y, en el caso urbano, amas de casa.

Es decir, un poco más del $60 \%$ de la población afirmó no tener idea de la democracia o que no le importaba. La encuesta mostró, además, las enormes brechas en conocimiento y actitudes, normalmente siguiendo las líneas de las desigualdades socioeconómicas, culturales y territoriales. Además, solo el $5 \%$ opinó que la democracia existía en el país y funcionaba bien, mientras que el 70,6\% reconocía que existía pero funcionando mal, y un cuarto de la población señaló que simplemente no vivíamos en democracia. El desconocimiento o desinterés de los ciudadanos no solo se refería a los grandes temas "nacionales", también se reflejaba en los espacios políticos más próximos: menos del $40 \%$ de los encuestados sabía el apellido del presidente de su región, 62 \% del alcalde provincial y $67 \%$ del alcalde distrital.

Según el estudio, una de las razones detrás de este desconocimiento y desinterés es que la democracia "formal" es una cuestión de minorías educadas y con altos ingresos económicos. Los pobres tienen su propia democracia cotidiana, que guarda poca o ninguna relación con la formal. La distancia entre lo "formal" y lo "vivencial" es uno de los factores que explica la ambivalencia con respecto a la democracia y las visiones que se tienen acerca de su práctica y viabilidad en el país. No nos debe llamar la atención, pues, que desde hace varios años, casi la mitad de los limeños y limeñas afirma que prefiere a un alcalde que roba pero que hace obras, a uno que no robe

9 El estudio sobre la democracia en el Perú, se basó en una muestra de 5200 hogares y 11116 entrevistas en 221 distritos de todos los departamentos (véase PNUD: La democracia en el Perú: el mensaje de las cifras, http://www.centrodesarrollohumano.org/pmb/opac_css/doc_num.php?explnum_id=745). 
pero haga pocas obras ${ }^{10}$. Pareciera que en el siglo XXI sigue teniendo vigencia el dictamen odriísta: "La democracia no se come".

En este, como en otros casos, las universidades podrían jugar un papel fundamental al generar puentes entre ciudadanos de todos los sectores e intentar conjugar el país formal con el informal. Los ciudadanos y ciudadanas reconocen este importante papel de la universidad, como bien se constata en la encuesta mencionada anteriormente realizada por la Universidad de Lima. Al ser preguntados si estaban de acuerdo con que las universidades promovieran el debate público, el 75,8 \% señalo que sí y el 21,7 \% que no. Las universidades deben aprovechar el hecho de que son confiables, esto potencia su capacidad de convocatoria, elemento de suma importancia en una sociedad que no confía más allá de la familia y las relaciones cercanas.

- Un segundo factor que facilita la confianza es que las personas perciban a los demás como competentes, hábiles y diestros. Cuando confiamos en un médico, abogado, arquitecto o ingeniero, no es necesariamente porque piense como nosotros, sino porque tiene las capacidades necesarias para sanarnos, defendernos, diseñar nuestro hogar o calcular correctamente las estructuras para construir nuestra casa, un puente o un sistema de saneamiento. Una parte esencial de la confianza es nuestra evaluación de la capacidad que tiene el otro para cumplir con nuestras expectativas. En este caso, la desconfianza no se da sobre la base del temor a que podríamos ser engañados, sino más bien a que la otra persona no tenga las habilidades para realizar lo que se espera de él o ella. Esta evaluación puede basarse en diversos elementos y puede incluir prejuicios, análisis de credenciales y experiencia, vivencias previas, entre otros.

Una de las debilidades cuando se intenta promover el desarrollo desde las bases sociales, es el nivel bajo de competencia -real o percibido- de las personas en tareas que son necesarias para impulsar el desarrollo. Esto incluye conocimientos técnicos, pero

10 Véanse las seis encuestas de opinión pública de la Pontificia Universidad Católica del Perú, realizadas durante el 2010, antes de las elecciones municipales. Ante la pregunta sobre el alcalde que roba pero hace obras, de 41 a $54 \%$ manifestaron preferirlo. http://iop.pucp.edu.pe/images/documentos/Intención $\% 20 \mathrm{de} \% 20$ voto\%20municipal\%20-\%20Setiembre\%202010\%203.pdf 
también de gestión de organizaciones, de participación ciudadana y política, de relación con otros sectores, de propuesta y cabildeo, entre otros. En situaciones normales en una comunidad tradicional, las competencias que se les exigían a dirigentes y comuneros eran aquellas que habían permitido el mantenimiento del status quo, a veces por períodos bastante largos. Sin embargo, si se apunta hacia el cambio social, el uso del capital social debe estar orientado hacia la búsqueda e implementación de formas más eficientes, productivas y sustentables de desarrollo, y esto amerita el surgimiento de nuevas competencias que, a su vez, deben ser reconocidas y apreciadas por los otros miembros de la comunidad.

En estos procesos, la educación y la capacitación juegan un papel esencial, especialmente cuando están acompañadas de la práctica y ofrecen al capacitado todo un mundo nuevo de relaciones, negociaciones, resolución de conflictos y otras actitudes democráticas o de liderazgo. Los capacitados, para convertirse en agentes catalizadores, como los llaman Uphoff y Wijayaratna (2000), deben ganarse la confianza de los integrantes de los grupos y las comunidades al demostrar que tienen los conocimientos y las destrezas necesarias para acompañar el proceso de cambio. Si la comunidad no confía en las competencias, percibe que el cambio es un riesgo demasiado grande. $\mathrm{O}$, por el otro lado, si no existen las competencias necesarias y se insiste en el cambio, los resultados tampoco van a ser beneficiosos. Estas son las lecciones aprendidas de proyectos de promoción del desarrollo.

Las universidades deben jugar un papel fundamental en el fortalecimiento de las capacidades de los ciudadanos del país. Esto no solamente al formar profesionales competentes y con valores éticos y de solidaridad, sino también contribuyendo a la formación de la ciudadanía en términos generales. Para ello, Vallaeys (2006) sugiere la constitución de "comunidades de aprendizaje", y recomienda:

La Universidad pasa convenios con actores externos (municipios, instituciones, asociaciones civiles, etc.) para crear programas solidarios mutuamente beneficiosos en el marco de Comunidades de Aprendizaje para el Desarrollo. La comunidad se desarrolla y la Universidad mejora su formación académica (estudiantil y docente) y produce nuevos conocimientos (investigación aplicada). (p. 19) 
- Un tercer factor que contribuye a la confianza es el nivel de cumplimiento de obligaciones mutuas. Como bien indica Durston (2000), la confianza siempre se construye en el pasado, y resulta de reiterados intercambios que fortalecen o debilitan la percepción de la responsabilidad de los demás. Es por ello que la confianza se edifica sobre la base de la cultura y la estructura social. Cultura, como hemos visto antes, al generar visiones de mundo comunes, en las cuales se comparten valores. Pero también es parte de la estructura, entendida en el sentido de cómo están ordenadas las relaciones sociales y las posibilidades de intercambios entre los diversos y diferentes.

La confianza nos motiva a acercarnos y a colaborar con los demás. Sin embargo, esta motivación debe fundamentarse en mecanismos estables que incentiven la continua colaboración y que sancionen o castiguen a los que no cumplen con las reglas básicas de cooperación. El grado de cumplimiento de estas reglas y la capacidad coercitiva de las sanciones son los principales determinantes de la efectividad de las normas. En términos de incentivos individuales, el nivel de efectividad de las normas es lo que determina los costos relativos de eventuales incumplimientos. A mayor efectividad, mayor será el costo de no cumplir, ya que las sanciones materiales, físicas y morales de una forma u otra impedirán o recortarán los beneficios resultantes de la participación en el grupo social. Una de las contribuciones de la conceptualización de capital social es hacer hincapié en que la capacidad de diseñar reglas y hacerlas cumplir (enforcement) es una de las medidas esenciales que facilita la acción colectiva y reduce el oportunismo en una sociedad. Las normas efectivas posibilitan el autocontrol en un conjunto humano, disminuyendo la necesidad de participación de terceros o agentes exógenos como garantes de las normas, sean contratos, obligaciones o derechos ${ }^{11}$.

11 Como bien ha señalado Putnam (1994), la presencia de terceros como garantes del cumplimiento de acuerdos, contratos y normas de reciprocidad no es una buena solución a los dilemas de la acción colectiva. Para ser efectiva, requeriría de terceros neutros, sin intereses o preferencias hacia ninguna de las partes, aspecto que no se puede garantizar. Esto genera el problema de quién controla y supervisa a los terceros, lo que lleva a una regresión infinita. 
Antes, cuando mostramos los niveles bajos de confianza, también indicamos que nuestros ciudadanos opinan que la mayoría no cumple las normas y que los derechos no son respetados. Esto significa que nuestra reputación como colectivo es bastante negativa. ¿Cómo promover entornos favorables para el cumplimiento de normas y sanciones efectivas? Hay varias maneras:

- Las universidades pueden fortalecer a las organizaciones encargadas de promover y hacer respetar las normas. En una primera instancia, lo pueden hacer en las mismas localidades en las cuales están ubicadas. Por ejemplo, desde el 2002 se han abierto enormes posibilidades de participación ciudadana en sus gobiernos locales y regionales. Los presupuestos participativos y los planes concertados de desarrollo son espacios concretos que necesitan de apoyo y asesoría técnica, de capacitaciones, estrategias de construcción de consensos y resolución de conflictos. Y, como señala Vallaeys (2006), al involucrarse la universidad, sus profesores y alumnos participan en procesos de enseñanza-aprendizaje que enriquecen a todos.

- Woolcock y Narayan (2000), expertos del Banco Mundial en el tema de capital social y desarrollo, recomiendan una visión "sinérgica" del capital social. Con esto se refieren a la necesaria participación de la comunidad, la empresa y el Estado en procesos conjuntos y compartidos de desarrollo. Cada una de las partes aporta sus ventajas comparativas. La comunidad aporta con su fuerza movilizadora, con la socialización en la familia y la vida en barrio, con las reglas cotidianas de convivencia, con la mirada más cercana a la problemática a ser trabajada. La empresa aporta organización y eficiencia, alternativas técnicas y económicas, la movilización de capital e inversión. El Estado aporta el imperio de la ley, la movilización de recursos necesarios para financiar bienes públicos, la prestación de servicios básicos, la socialización vía la escuela, entre otros. ¿Y la universidad? Bueno, puede ser el pegamento que junta a estos actores, ya que tiene contacto estrecho y experiencia de trabajo con todos ellos. Su principal ventaja es que sigue siendo un actor confiable y solicitado.

- Nuestro país, como hemos mencionado anteriormente, se caracteriza por tejer redes sociales cercanas, es decir, relaciones socia- 
les entre personas conocidas. Todo ello, como producto de nuestra desconfianza y la búsqueda de la familia y los amigos como refugio ante instituciones inoperantes o corruptas. Este ensimismamiento lleva a que nuestros vínculos sociales tiendan a ser del tipo que "une" (bonding, en inglés). Sin embargo, estos vínculos que protegen también nos aíslan y no nos permiten acceder a los recursos de los demás, de los terceros, de los lejanos. Para acceder se necesita un segundo tipo de vínculo, denominado "de puente" (bridging), el cual nos permite acceder a recursos más allá de los cercanos y familiares. La información, por ejemplo, es uno de los recursos básicos a los que accedemos desde nuestras redes. Cuando una red es aislada, la información no es novedosa y es redundante. Cuando una red tiende puentes, nos abrimos a un mundo de información novedosa y transformadora.

En nuestro país fragmentado y de brechas sociales, los pobres son los que cuentan con menos vínculos "de puente" y no pueden acceder a los beneficios de una red más amplia. Consideramos que la universidad puede ayudar a construir ese puente, al igual que lo han hecho las organizaciones no gubernamentales (ONG) desde hace varias décadas. El contacto con los programas estatales, la asesoría profesional, los fondos de la cooperación internacional, mercados nuevos, información útil y pertinente, todos nacen de relaciones puente.

Con estas breves sugerencias y ejemplos, he tratado de explicar cómo la universidad puede "palanquear" la confianza que goza -en un país de desconfiados- para promover procesos que fortalecen los mecanismos de cooperación y colaboración entre los peruanos. Las universidades pueden mejorar y enriquecer las experiencias de trabajo conjunto entre el Estado y la población en la mira de proyectos de coproducción; pueden asesorar la elaboración de presupuestos participativos realistas y apoyar el seguimiento y la vigilancia de su correcta ejecución; deben elevar el nivel de los debates políticos y públicos al fiscalizar la idoneidad de las propuestas y al exigir soluciones eficientes y eficaces para los problemas de nuestras localidades y regiones; deben dar el ejemplo en la gestión sostenible impulsando programas de ahorro de energía en sus campus, reduciendo desechos y reciclando e impulsando mecanismos para replicar estas experiencias en la comunidad. En fin, todo un enorme abanico de acciones que, al generar soluciones, van fortaleciendo 
nuestra institucionalidad democrática y mostrando que la democracia también da de comer, que brinda salud y construye estabilidad. Todo ello, dentro de una concepción ética de responsabilidad hacia las sociedades que nos albergan y sustentan.

\section{Referencias}

Coleman, J.S. (1994). Foundations of social theory. Cambridge: Harvard University Press.

Comisión de las Comunidades Europeas. (2001). Libro verde: fomentar un marco europeo para la responsabilidad social de las empresas. Bruselas: CCE.

Díaz-Albertini, J. (2003). Capital social, organizaciones de base y el Estado: Recuperando los eslabones perdidos de la sociabilidad. Capital social y reducción de la pobreza en América Latina y el Caribe: en busca de un nuevo paradigma. Santiago de Chile: Comisión Económica para América Latina y el Caribe/Michigan State University, pp. 247-302.

Díaz-Albertini, J. (2010). Redes cercanas: el capital social en Lima. Universidad de Lima.

Durston, J. (diciembre, 1999). Construyendo capital social comunitario. Revista de la Cepal, 69, pp. 103-118.

Durston, J. (2000). ¿Qué es el capital social comunitario? Santiago de Chile: Cepal, División de Desarrollo Social, Serie Políticas Sociales, n. ${ }^{0} 38$.

Evans, P. (1996). Government Action, Social Capital and Development: Reviewing the Evidence on Synergy. World Development, 24 (6), pp. 1119-1132.

Fukuyama, F. (1996). Confianza (Trust). Buenos Aires: Editorial Atlántida.

Hansmann, H. (2003). The Role of Trust in the Nonprofit Enterprise. En H.K. Anheier, A. Ben-Ner (Eds.), The Study of Nonprofit Enterprise. Nueva York: Springer, pp. 115-122.

Holme, R., \& Watts, P. (2000). Corporate Social Responsibility: Making good business sense. Ginebra: World Business Council for Sustainable Development.

Knack, S., \& Keefer, P. (1997). Does Social Capital Have an Economic Payoff? A Cross-Country Investigation. Quarterly Journal of Economics, 112 (4), pp. 1251-1288. 
Latinobarómetro. (2012). Banco de datos. Recuperado de: www.latino barómetro.org

Latinobarómetro. (2011). Banco de datos. Recuperado de: www.latino barómetro.org

Latinobarómetro. (2011). Banco de datos. Recuperado de: www.latino barómetro.org

Portes, A. (2000). Social Capital: Its Origins and Applications in Modern Sociology. En E. Lesser (Ed.), Knowledge and Social Capital. Boston: Butterworth-Heinenmann, pp. 43-67.

Portocarrero, F. (2000). Empresas, fundaciones y medios: la responsabilidad social en el Perú. Lima: Universidad del Pacífico.

Programa de las Naciones Unidas para el Desarrollo-PNUD. (2006). La democracia en el Perú: el mensaje de las cifras. Lima: Autor.

Putnam, R.D., Leonardi, R., \& Nanetti, R.Y. (1994). Making Democracy Work, Civic Traditions in Modern Italy. Princeton: Princeton University Press, 1994.

Rama, C. (2006). Introducción: la tercera reforma de la educación superior en América Latina y el Caribe: masificación, regulación e internacionalización. Informe sobre la educación superior en América Latina y el Caribe 2000-2005: La metamorfosis de la educación superior. Caracas: Instituto Internacional de la Unesco para la Educación Superior en América Latina y el Caribe (IESALC), pp. 11-18.

Rose-Ackerman, S. (junio 1996). Altruism, Nonprofits, and Economic Theory. Journal of Economic Literature (vol. XXXXIV), pp. 701-728.

Universidad de Lima, Grupo de Opinión Pública. (marzo 2007). II Encuesta sobre la situación de la universidad peruana, estudio 363, barómetro social.

Uphoff, N. \& Wijayaratna, C.M. (noviembre 2000). Demonstrated Benefits from Social Capital: The Productivity of Farmer Organizations in Gal Oya, Sri Lanka. World Development, 28 (11), pp. 1875-1890.

Vallaeys, F. (2006). La responsabilidad social universitaria. Lima: Pontificia Universidad Católica del Perú.

Vallaeys, F., De La Cruz, C., \& Sasia, P. (2009). Responsabilidad social universitaria: manual de primeros pasos. México: McGraw Hill Interamericana.

Wallerstein, I. (1996). Abrir las ciencias sociales. Madrid: Siglo XXI. 
Woolcock, M., \& Narayan, D. (agosto 2000). Social Capital: Implications for Development Theory, Research, and Policy. The World Bank Research Observer, 15 (2), pp. 225-249.

Yamada, G. (2007). Retornos a la educación superior en el mercado laboral: ¿Vale la pena el esfuerzo? Lima: Centro de Investigación de la Universidad del Pacífico y Consorcio de Investigación Económica y Social, Documento de Trabajo 78. 